\title{
Objective Structured Clinical Examination in Intensive Care Medicine
}

\author{
Jeyasankar Jeyanathan, Daniel Owens, Nikki Bramhill (editors). tfm Publishing Limited, \\ 2016. ISBN 978-1-910079-23-2
}

Asher Aron Mendelson, MDCM

Received: 13 March 2016/Revised: 24 March 2016/Accepted: 15 April 2016/Published online: 26 April 2016

(c) Canadian Anesthesiologists' Society 2016

The Objective Structured Clinical Examination (OSCE) in medical education is designed to evaluate the ability of candidates to synthesize clinical information quickly and accurately and to outline a hypothesis and management plan. Successful candidates are able to construct organized, thorough responses effectively in a professional, reassuring communication style. Although each OSCE station is typically less than $15 \mathrm{~min}$ in duration, examiners are able to assess candidates reliably for competence and suitability for independent medical practice. Increasingly, medical colleges are incorporating the OSCE into the credentialing process, using it as an adjunct to the written licensing examination.

Objective Structured Clinical Examination in Intensive Care Medicine provides trainees in critical care a fastreading, straightforward reference book as they prepare for their board examinations. Intended as a study guide, the book is easy to read. The font is large, and the bullet point format of the text keeps distractions to a minimum. Clinical images, laboratory results, clinical scoring systems, and take-home points are effectively interspersed to engage the reader. This book provides insight into the style of OSCE questions and examples of how trainees are scored for various responses. The book includes common OSCE stations for clinical topics frequently encountered in the intensive care unit (ICU) and provides a framework for responding to questions by the examiner.

Emphasis is placed on several key OSCE themes, including (1) succinct interpretation of laboratory tests and

A. A. Mendelson, MDCM ( $\varangle)$

Division of Critical Care, Schulich School of Medicine and Dentistry, University of Western Ontario, London, ON, Canada

e-mail: asher.mendelson@mail.mcgill.ca diagnostic imaging, (2) broad generation of a differential diagnosis, (3) prompt initiation of key management interventions, (4) appropriate follow-up investigations, and (5) disease-specific knowledge and content expertise. Starting each OSCE station with a clinical stem, as well as inclusion of dedicated OSCE stations aimed at addressing professionalism/communication and ICU equipment/ monitoring, make this book relevant to the contemporary OSCE examinations currently offered by licensing boards.

This book is not designed as a primary source textbook but, rather, an outline for trainees who are sharpening their OSCE technique prior to the examination. There are still instances within this context, however, where the book does not provide sufficient information or rationale to explain recommended investigations and management decisions. This approach perhaps aims to reinforce the need for focused responses during the OSCE that do not embellish or become heavy with excessive detail. It may become problematic for trainees, however, if they are then asked to address follow-up questions from examiners.

Rather than grouping topics according to disease or organ system, the book is divided into five chapters containing OSCE stations from across the broad spectrum of critical care medicine. This arrangement appears to have been designed to simulate the format of the OSCE, but it becomes confusing and often does not flow well. This approach also causes splitting and reduplication of content throughout the book that often prevents focused reading. Moreover, subtopics become embedded in stations with loosely related themes, making it difficult to review or reference these subtopics at a later date.

Even more concerning is the inclusion of several factually incorrect statements that challenge the credibility of the book as a medical reference. For 
example, the authors incorrectly state that rapid correction of hypernatremia is associated with a risk of central pontine myelinolysis (p. 30), which of course is a complication of rapid hyponatremia correction. They also state that bilateral interstitial changes and traction bronchiectasis are features of chronic obstructive pulmonary disease (p. 115) when they are actually associated with idiopathic pulmonary fibrosis. Another error relates to their asssertion that treatment with intravenous immunoglobulin is currently recommended for Staphylococcus necrotizing pneumonia (p. 164) when it is better reserved for Streptococcus pyogenes infection. Lastly, they state that insertion of a Sengstaken-Blakmore tube through either the nose or mouth is acceptable (p. 231) whereas oral insertion is, by far, the preferred method.

One of the innovative features of this book is the attention given to recent landmark trials that have shaped practice in key areas of critical care. Therapeutic advances in the treatment of acute respiratory distress syndrome are highlighted, and current evidence regarding fluid management and early goal-direct therapy for septic shock are discussed. This approach, however, is not consistently applied throughout the book, with key trials not being mentioned in other sections. Consequently, certain recommendations such as prescribing steroids for pneumonia (p. 21) and decompressive craniectomy for traumatic brain injury (p. 260) appear to carry more authority than is afforded them in the best available literature. These assertions may expose trainees to hostile lines of questioning from examiners unless they are framed within the context of current evidence-based practice.

This book succeeds in familiarizing readers with the style and format they can expect from an OSCE in intensive care medicine. It can be read quickly and reinforces the salient, structured approach required to prepare for an oral certification examination. From a medical reference standpoint, however, its content could be better organized and is often treated superficially and inconsistently. As a result, reading this book requires meticulous cross-referencing and supplementation from various other source texts and may leave the reader with more questions than would be expected. This book should be considered a supplement for intensive care medicine trainees wishing to place particular focus on improving their OSCE skills and for program directors who wish to incorporate OSCE-based learning in their curriculum.

Conflicts of interest None declared.

Editorial responsibility This submission was handled by Dr. Hilary P. Grocott, Editor-in-Chief, Canadian Journal of Anesthesia. 